\title{
Dynamical generation of resonances in the $P 33$ partial wave
}

\author{
B. Golli $\odot^{*}$ \\ Faculty of Education, University of Ljubljana, 1000 Ljubljana, Slovenia \\ and J. Stefan Institute, 1000 Ljubljana, Slovenia \\ H. Osmanović $(1)$ \\ Faculty of Natural Sciences and Mathematics, University of Tuzla 75000 Tuzla, Bosnia and Hercegovina \\ S. Širca $\odot$ \\ Faculty of Mathematics and Physics, University of Ljubljana, 1000 Ljubljana, Slovenia \\ and J. Stefan Institute, 1000 Ljubljana, Slovenia
}

(Received 9 July 2019; published 19 September 2019)

\begin{abstract}
We investigate the formation of resonances in the $P 33$ partial wave with emphasis on possible emergence of dynamically generated quasibound states as a consequence of a strong $p$-wave pion attractive interaction in this partial wave, as well as their possible interaction with the genuine quark excited states. By using the LaurentPietarinen expansion we follow the evolution of the $S$-matrix poles in the complex energy plane as a function of the interaction strength. Already without introducing a genuine quark resonant state, two physically interesting resonances emerge with pole masses around 1200 and $1400 \mathrm{MeV}$, with dominant $\pi N$ and $\pi \Delta$ components, respectively. The added genuine resonant state in the $(1 s)^{3}$ quark configuration mixes with the lower dynamically generated resonance forming the physical $\Delta(1232)$ resonance, and pushes the second dynamical resonance to around $1500 \mathrm{MeV}$, which allows it to be identified with the $\Delta(1600)$ resonance. Adding a second resonant state with one quark promoted to the $2 s$ orbit generates another pole whose evolution remains well separated from the lower two poles. We calculate the helicity amplitudes at the pole and suggest that their $Q^{2}$ dependence could be a decisive test to discriminate between different models of the $\Delta(1600)$ resonance.
\end{abstract}

DOI: 10.1103/PhysRevC.100.035204

\section{INTRODUCTION}

Since its discovery in 1952 [1,2], the $\Delta(1232)$ resonance has played a significant role in almost all attempts to understand the structure and dynamics of the nucleon and its excited states. The early approaches were based on the observation that the dominant $p$-wave pion nucleon interaction is attractive in the $P 33$ partial wave - in contrast to the $P 11$, $P 13$, and $P 31$ waves - and may therefore generate a resonance at the observed energy, provided that the coupling is sufficiently strong. Since the pion-nucleon coupling constant was known from the behavior of the $P 11$ wave near the threshold, the above condition required a sufficiently large cutoff, of the order of $1 \mathrm{GeV} / c$ [3]. With the introduction of the quark model, the four charge states of $\Delta(1232)$ have been identified as the isospin quadruplet belonging to the

\footnotetext{
*bojan.golli@ijs.si
}

Published by the American Physical Society under the terms of the Creative Commons Attribution 4.0 International license. Further distribution of this work must maintain attribution to the author $(s)$ and the published article's title, journal citation, and DOI. Funded by $S C O A P^{3}$. lowest quark decuplet. The excitation energy with respect to the nucleon is usually explained by the gluon and/or pion exchange interaction between quarks. The relatively strong $p$-wave pion-nucleon interaction-though not the main mechanism generating the resonance-manifests itself in sizable pion contributions to the photo- and electroproduction amplitudes.

While the properties of the $\Delta(1232)$ resonance are well understood, this is not the case with the next higher resonance in the $P 33$ partial wave, the $\Delta(1600)$. In the quark model, this resonance is traditionally described as the radial excitation in which one quark is promoted to the $2 s$ orbit; the analog to the Roper resonance $N(1440)$ in the $P 11$ partial wave. The problem with such an interpretation is that in the harmonicoscillator model the $2 s$ excitation is twice as large as the $1 p$ excitation while the observed $N(1440)$ resonance appears below the negative parity resonances. Furthermore, recent results of lattice QCD in the $P 11$ partial wave show no clear signal for a three-quark Roper state below $1.7 \mathrm{GeV}[4,5]$. To resolve the problem of level ordering, an alternative approach has been proposed in which coupled-channel meson-baryon dynamics alone was sufficient to engender the resonance [6,7]. In our previous work [8] we have shown that, while the mass of the $N(1440)$ resonance is indeed determined by the dynamically generated state with the dominant $s$-wave $\sigma N$ 
component, a genuine three-quark $(1 s)^{2} 2 s$ component with mass above $1750 \mathrm{MeV}$ is needed to explain the properties of the resonance. The presence of a bare baryon structure at around $1750 \mathrm{MeV}$ has also been emphasized in the Excited Baryon Analysis Center approach $[9,10]$.

Since the $\Delta(1600)$ resonance may be considered as a spin $\frac{3}{2}$, isospin $\frac{3}{2}$ partner of the $N(1440)$, it seems at first glance that a similar model could work also in the $P 33$ partial wave with the $\sigma N$ substituted by the $s$-wave $\sigma \Delta$ component. ${ }^{1}$ As shown in [8] the $N(1440)$ mass at the pole lies slightly below the nominal $N \sigma$ threshold, rather independently of the model parameters, which would mean that the mass of the $\Delta(1600)$ would be at least $200 \mathrm{MeV}$ higher than the mass of the pole given by the Particle Data Group (PDG) [11], ruling out such a model. Also, a preliminary calculation in the $P 33$ partial wave has shown that the $\sigma \Delta$ component represents a rather minor contribution to the scattering amplitudes below $W \approx 1800 \mathrm{MeV}$. We therefore consider here an alternative model, based on the observation that the $\pi N$ as well as the $\pi \Delta$ interaction are attractive in the $P 33$ partial wave, in which the dynamically generated state consists of quasibound $\pi N$ and $\pi \Delta$ states. Such a model is further stimulated by the study [12] using a semiphenomenological approach to extract photoproduction couplings at the pole of $N$ and $\Delta$ resonances up to $W \approx 2.4 \mathrm{GeV}$, which has confirmed the dynamical origin of the $\Delta(1600)$ resonance with a dominant $\pi \Delta$ configuration.

There have not been many attempts to study the properties of $\Delta(1600)$ from the quark modeling point of view; let us mention the calculations in the relativistic quark model in a light-front framework [13-15] assuming the dominant $(1 s)^{2} 2 s$ quark configuration, which leads to a similar behavior of the helicity amplitude as in the Roper case, and a calculation [16] using a relativistic diquark-quark model.

In the next section we briefly review the basic features of our coupled-channels approach and of the underlying quark model that has been used in our treatment of $N(1440)$. However, in the version reported here we do not include the $\sigma$ meson, which turns out to have only a very minor role in the relevant energy region. Furthermore, since the $\pi N N$ coupling constant is well established, we keep the $\pi$ quark coupling constant fixed and vary the cutoff parameter in order to study the evolution of the resonance poles in the complex energy plane by using the Laurent-Pietarinen $(\mathrm{L}+\mathrm{P})$ expansion [17-20]. In Sec. III we solve the coupledchannels problem, first without including any genuine threequark resonant state, then by including a three-quark resonant state corresponding to $\Delta(1232)$, and finally adding a threequark resonant state in a $(1 s)^{2} 2 s$ configuration. In Sec. IV we discuss the prediction of our model for the photo- and electroproduction amplitudes which may eventually support our picture of the $\Delta(1600)$.

\footnotetext{
${ }^{1}$ In the following we shall denote the $\Delta(1232)$ as $\Delta ;$ the $\Delta(1600)$ will be eventually denoted as $\Delta^{*}$.
}

\section{THE MODEL}

In our approach the scattering state in channel $\alpha$ which includes a quasibound quark state $\Phi_{R}$ assumes the form

$$
\begin{aligned}
\left|\Psi_{\alpha}\right\rangle= & \mathcal{N}_{\alpha}\left\{\left[a_{\alpha}^{\dagger}\left(k_{\alpha}\right)\left|\Phi_{\alpha}\right\rangle\right]+c_{\alpha R}\left|\Phi_{R}\right\rangle\right. \\
& \left.+\sum_{\beta} \int \frac{d k \chi_{\alpha \beta}\left(k_{\alpha}, k\right)}{\omega_{\beta}(k)+E_{\beta}(k)-W}\left[a_{\beta}^{\dagger}(k)\left|\Phi_{\beta}\right\rangle\right]\right\},
\end{aligned}
$$

where $\alpha(\beta)$ denotes either $\pi N$ or $\pi \Delta$ channels and [ ] stands for coupling to total spin $\frac{3}{2}$ and isospin $\frac{3}{2}$. The first term represents the free pion and the baryon ( $N$ or $\Delta)$ and defines the channel, the next term corresponds to a bare three-quark resonant state, while the third term describes the pion cloud around the nucleon and $\Delta$. Here $\mathcal{N}_{\alpha}=\sqrt{\omega_{\alpha} E_{\alpha} /\left(k_{\alpha} W\right)}, k_{\alpha}$ and $\omega_{\alpha}$ are on-shell pion momentum and energy, and $W=$ $\omega_{\alpha}+E_{\alpha}$ is the invariant mass. The integral is assumed in the principal value sense. The (half-on-shell) $K$ matrix is related to the scattering state as [21]

$$
K_{\alpha \beta}\left(k_{\alpha}, k\right)=-\pi \mathcal{N}_{\beta}\left\langle\Psi_{\alpha}\left\|V^{\beta}(k)\right\| \Phi_{\beta}\right\rangle,
$$

with the property $K_{\alpha \beta}\left(k_{\alpha}, k\right)=K_{\beta \alpha}\left(k, k_{\alpha}\right)$. It is proportional to the pion amplitude $\chi$ in (1),

$$
K_{\alpha \beta}\left(k_{\alpha}, k\right)=\pi \mathcal{N}_{\alpha} \mathcal{N}_{\beta} \chi_{\alpha \beta}\left(k_{\alpha}, k\right) .
$$

The amplitude $\chi$ satisfies a Lippmann-Schwinger type of equation:

$$
\begin{aligned}
\chi_{\alpha \gamma}\left(k, k_{\gamma}\right)= & -c_{\gamma R} V_{\alpha R}(k)+\mathcal{K}_{\alpha \gamma}\left(k, k_{\gamma}\right) \\
& +\sum_{\beta} \int d k^{\prime} \frac{\mathcal{K}_{\alpha \beta}\left(k, k^{\prime}\right) \chi_{\beta \gamma}\left(k^{\prime}, k_{\gamma}\right)}{\omega_{\beta}\left(k^{\prime}\right)+E_{\beta}\left(k^{\prime}\right)-W} .
\end{aligned}
$$

Our model utilizes two approximations for the kernel $\mathcal{K}$ : the first one implies only $u$-channel processes,

$$
\mathcal{K}_{\alpha \beta}\left(k, k^{\prime}\right)=\sum_{i=N, \Delta} f_{\alpha \beta}^{i} \frac{V_{i \beta}^{\alpha}(k) V_{i \alpha}^{\beta}\left(k^{\prime}\right)}{\omega_{\alpha}(k)+\omega_{\beta}\left(k^{\prime}\right)+E_{i}(\bar{k})-W},
$$

and the second one implies that the kernel can be made separable by assuming

$$
\begin{aligned}
& \frac{1}{\omega_{\alpha}(k)+\omega_{\beta}\left(k^{\prime}\right)+E_{i}-W} \\
& \quad \approx \frac{\left(\omega_{\alpha}+\omega_{\beta}+E_{i}-W\right)}{\left[\omega_{\alpha}(k)+E_{i}-E_{\beta}\right]\left[\omega_{\beta}\left(k^{\prime}\right)+E_{i}-E_{\alpha}\right]},
\end{aligned}
$$

where $W=E_{\alpha}+\omega_{\alpha}=E_{\beta}+\omega_{\beta}$. The factorization is exact if either of the $\omega$ 's is on shell, i.e., $\omega_{\alpha}(k) \rightarrow \omega_{\alpha}=W-E_{\alpha}$ or $\omega_{\beta}\left(k^{\prime}\right) \rightarrow \omega_{\beta}=W-E_{\beta}$. In the present work we include only pion loops and the nucleon and $\Delta$ as the $u$-channel exchange particles. Based on our previous experience in the $P 11$ and $P 33$ partial waves these degrees of freedom dominate in the energy region considered in the following. The spin-isospin 
factors in (5) equal

$$
\begin{aligned}
& f_{N N}^{N}=f_{N N}^{\Delta}=\frac{4}{9}, \quad f_{N N}^{\Delta}=\frac{1}{36}, \quad f_{\Delta \Delta}^{\Delta}=\frac{121}{225}, \\
& f_{N \Delta}^{N}=f_{\Delta N}^{N}=\frac{5}{9}, \quad f_{N \Delta}^{\Delta}=f_{\Delta N}^{\Delta}=\frac{2}{9} .
\end{aligned}
$$

Equation (5) implies dressed vertices; in the present calculation the vertices involving the $\Delta$ are increased by $30 \%$ with respect to their bare (quark model) values, in accordance with our analysis of the $P 33$ resonances in [21], while $V_{\pi N N}$ is kept at its bare value.

The vertices are determined in the underlying quark model, which can be chosen freely; we use the cloudy bag model [22], which involves two parameters: the pion-decay constant, $f_{\pi}$ (reduced to $76 \mathrm{MeV}$ in order to reproduce the $\pi N$ coupling constant), and the bag radius which determines the cutoff. In our previous analysis we used a typical value of $R=$ $0.83 \mathrm{fm}$ corresponding to the cutoff $\Lambda \approx 550 \mathrm{MeV}$. These two parameters describe consistently the scattering and photoproduction amplitudes, including the production of $\eta$ and $K$ mesons $[8,21,23-26]$. In order to reveal the mechanism of $\Delta(1600)$ formation we study the evolution of the resonance properties as a function of $R$ (which is inversely proportional to the cutoff momentum) for a large range of its value, keeping in mind that the physically sensible interval should be between 0.6 and $1 \mathrm{fm}$.

The pion amplitude can be written in terms of the resonant and nonresonant parts,

$$
\chi_{\alpha \gamma}\left(k, k_{\gamma}\right)=c_{\gamma R} \mathcal{V}_{\alpha R}(k)+\mathcal{D}_{\alpha \gamma}\left(k, k_{\gamma}\right),
$$

such that (4) can be split into the equation for the dressed vertex,

$$
\mathcal{V}_{\alpha R}(k)=V_{\alpha R}(k)+\sum_{\beta} \int d k^{\prime} \frac{\mathcal{K}_{\alpha \beta}\left(k, k^{\prime}\right) \mathcal{V}_{\beta R}\left(k^{\prime}\right)}{\omega_{\beta}\left(k^{\prime}\right)+E_{\beta}\left(k^{\prime}\right)-W},
$$

and the nonresonant amplitude,

$$
\mathcal{D}_{\alpha \gamma}\left(k, k_{\gamma}\right)=\mathcal{K}_{\alpha \gamma}\left(k, k_{\gamma}\right)+\sum_{\beta} \int d k^{\prime} \frac{\mathcal{K}_{\alpha \beta}\left(k, k^{\prime}\right) \mathcal{D}_{\beta \gamma}\left(k^{\prime}, k_{\gamma}\right)}{\omega_{\beta}\left(k^{\prime}\right)+E_{\beta}\left(k^{\prime}\right)-W},
$$

with

$$
c_{\alpha R}=-\frac{\mathcal{V}_{\alpha R}\left(k_{\alpha}\right)}{W-m_{R}+\sum_{\beta} \int d k \frac{\mathcal{V}_{\beta R}(k) V_{\beta R}(k)}{\omega_{\beta}(k)+E_{\beta}(k)-W}},
$$

where $m_{R}$ is the bare mass of the resonant state. ${ }^{2}$

Since the kernel (5) has been rendered separable, equations (8) and (9) can be solved exactly (i.e., to all orders) with the Ansätze

$$
\mathcal{V}_{\alpha R}(k)=V_{\alpha R}(k)+\sum_{\beta i} x_{\beta i}^{\alpha} \varphi_{\beta i}^{\alpha}(k)
$$

and

$$
\mathcal{D}_{\alpha \gamma}\left(k, k_{\gamma}\right)=\mathcal{K}_{\alpha \gamma}\left(k, k_{\gamma}\right)+\sum_{\beta i} z_{\beta i}^{\alpha \gamma} \varphi_{\beta i}^{\alpha}(k)
$$

\footnotetext{
${ }^{2}$ Equation (10) becomes more complicated if the second resonant state is included; see Sec. III C.
}

where

$$
\begin{aligned}
\varphi_{\beta i}^{\alpha}(k) & =\frac{2 m_{i}}{E_{\beta}}\left(\omega_{\beta}+\varepsilon_{i \alpha}^{\beta}\right) \frac{V_{i \beta}^{\alpha}(k)}{\omega_{\alpha}(k)+\varepsilon_{i \beta}^{\alpha}} f_{\alpha \beta}^{i}, \\
\varepsilon_{i \alpha}^{\beta} & =\frac{m_{i}^{2}-m_{\alpha}^{2}-\mu_{\beta}^{2}}{2 E_{\alpha}} .
\end{aligned}
$$

This leads to a set of linear algebraic equations for the coefficients $x$ :

$$
\sum_{\gamma j} A_{\alpha i, \gamma j}^{\beta} x_{\gamma j}^{\beta}=b_{\alpha i}^{\beta},
$$

and similarly for $z$ with the same $A$ matrix. Here $i(j)$ refers to $u$-channel exchange baryons ( $N$ and $\Delta)$. The expression for the $A$ matrix along with its graphical representation as well as the right-hand side's $b$ are given in [8]. ${ }^{3}$ Having obtained the $K$ matrix, the scattering matrix $T$ is obtained by solving the Heitler equation.

With increasing interaction strength the kernel may become singular, and the $K$ matrix acquires a pole which may be interpreted as a dynamically generated resonance. In [8] we performed the singular value decomposition of the $A$ matrix [27] in order to be able to determine the $W$ dependence of the lowest singular value, $w_{\min }$, and to study how the behavior of $w_{\min }$ is reflected in the evolution of pole(s) in the complex plane as the interaction strength is increased. The main conclusion of such an analysis in our previous work has been that the pole corresponding to the dynamically generated resonance emerges well before the interaction strength reaches the value at which $w_{\min }$ touches zero. Furthermore, the mass of the pole turns out to lie close to the position where $w_{\min }$ reaches its minimum, almost independently of the interaction strength. This property persists even when $w_{\min }$ becomes negative; however, additional poles may show up at $W$ where $w_{\min }$ crosses zero. From the corresponding eigenvectors it is possible to establish the main meson-baryon components of the dynamically generated state.

\section{SOLVING THE SCATTERING EQUATION}

\section{A. No bare-baryon resonant state}

We first study the case without any genuine bare baryon, so the problem reduces to solving Eq. (9) alone. We consider two cases: in the first one we assume only the nucleon as the $u$ channel exchange particle and fix the $\pi N N$ coupling constant to the experimental value; in the second case we add the $\Delta$ at $1232 \mathrm{MeV}$ as the second $u$-channel exchange particle and fix the $\pi \Delta N$ and the $\pi \Delta \Delta$ coupling constants to the quark-model values increased by $30 \%$. We vary the coupling strength by changing the bag radius. Figure 1 shows the behavior of $w_{\text {min }}$ as a function of $W$ for some typical values of $R$ for the two cases. In the first case (thinner curves) $w_{\text {min }}$ touches zero for $R \approx 0.22 \mathrm{fm}$ and crosses zero at $1232 \mathrm{MeV}$ for $R=0.123 \mathrm{fm}$. The situation is considerably more complex in the second case (thick curves). For the physically interesting values around $R=0.8 \mathrm{fm}, w_{\min }$ exhibits three minima at around 1200,1500 ,

\footnotetext{
${ }^{3}$ In the present model the $\sigma$ terms are not included.
} 


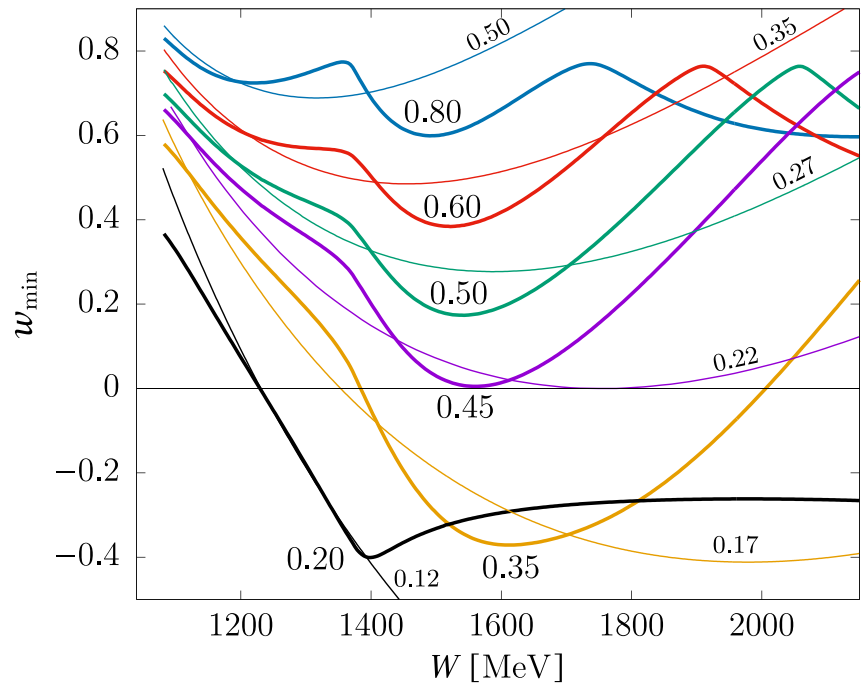

FIG. 1. The behavior of $w_{\min }$, the lowest singular value of $A$ as a function of $W$ for the kernel involving $N$ and $\Delta u$-channel exchange (thick lines) and $N$ alone (thin lines), for different bag radii $R$ (in fm).

and $2000 \mathrm{MeV}$. For smaller $R$ the middle minimum stays close to $1500 \mathrm{MeV}$ and touches zero for $R \approx 0.45 \mathrm{fm}$. The zero crossing at $1232 \mathrm{MeV}$ occurs for $R=0.20 \mathrm{fm}$.

In order to obtain the scattering amplitudes we have to specify how to include the inelastic channel above the twopion threshold. We assume that the decay into two pions proceeds through the $\pi \Delta$ intermediate state as described in [23] and in Appendix A of [24], which implies an integration over the invariant mass of the $\pi N$ system weighted by the probability determined in the $\pi N$ scattering in the $P 33$ partial wave. We assume that this probability is given by the BreitWigner mass and width of the $\Delta$. As we shall see in the following, this assumption is consistent for sufficiently strong coupling (small $R$ ) where the parameters of the $\Delta(1232)$ are reproduced in the same dynamical model.

The resulting scattering amplitudes are displayed in Fig. 2 for three typical bag radii. While for larger values of $R$ the amplitudes do not show any visible sign of resonance, for $R=$ $0.123 \mathrm{fm}$ (for the $u$-channel $N$-exchange kernel) and for $R=$ $0.20 \mathrm{fm}$ ( $N$ and $\Delta$ exchange) they perfectly fit the experimental data below $1300 \mathrm{MeV}$. By using the $\mathrm{L}+\mathrm{P}$ expansion we have been able to follow the evolution of the pole(s) in the two cases considered above from the (relatively) weak coupling towards the strong coupling regime. Using the kernel with solely the $N$ exchange there is only one resonance which can be attributed to the dynamically generated $\Delta(1232)$. It starts far from the real axis (i.e., with a large width), approaches the real axis, and becomes bound for $R=0.050 \mathrm{fm}$ (see Fig. 3). It is interesting to notice that its pole mass remains close to $1200 \mathrm{MeV}$. For $R=0.123 \mathrm{fm}$ where $\operatorname{Re} T$ reaches zero at $1232 \mathrm{MeV}$, the pole parameters agree well with those extracted from experiments (see Table I).

The situation in the case with $N$ and $\Delta$ exchange particles is much more complex: for larger $R$ there are two poles, and, for the radius for which $w_{\text {min }}$ comes close to zero, a third pole emerges. The lowest one exhibits a very similar

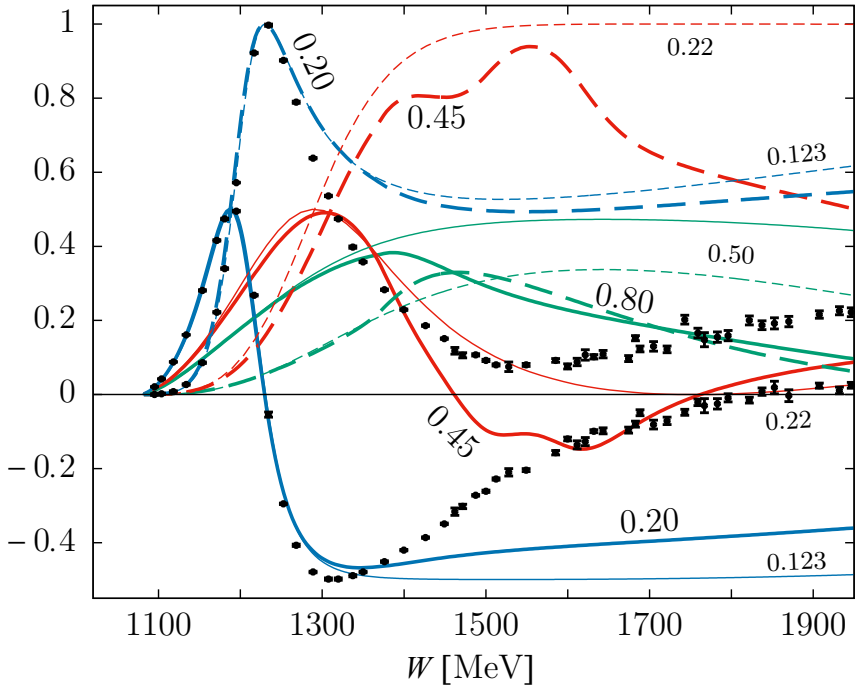

FIG. 2. $T$-matrix amplitudes involving $N$ and $\Delta u$-channel exchange (thick lines, dashes for $\operatorname{Im} T$ ) for $R=0.8 \mathrm{fm}, 0.45$ and $0.20 \mathrm{fm}$, and $N$ alone (thin lines, dashes for $\operatorname{Im} T$ ) for $R=0.5,0.22$, and $0.123 \mathrm{fm}$. Experimental data are from [28,29].

evolution as the pole in the previous case, while the second pole remains close to the mass $1380 \mathrm{MeV}$ and width of $220 \mathrm{MeV}$, rather independently of $R$. Since these two poles lie close to each other, their determination is not very precise; the unsmooth evolution curve in the complex plane can be therefore attributed to numerical instabilities. The second pole turns out to be better determined in the $\pi \Delta$ channel (see Table I). This pole can be interpreted as the progenitor of the $\Delta(1600)$ resonance. For smaller $R$ the mass of the third pole coincides with the energy at which $w_{\min }$ crosses zero the second time; it seems to have no physical interpretation and might be an artifact of the model.

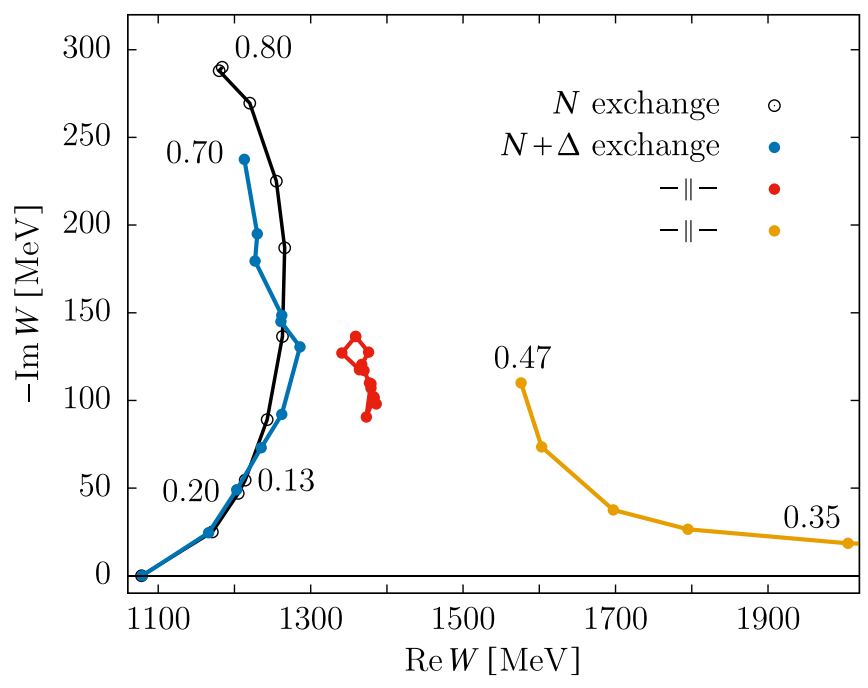

FIG. 3. Evolution of poles in the complex plane as a function of $R$ (in fm): the single pole arising from the $u$-channel $N$-exchange kernel (open circles), and the three poles from the $N+\Delta$ exchange kernel (full circles). 
TABLE I. $S$-matrix pole position, modulus, and phase for the $u$-channel $N$-exchange (I) and $\Delta+N$-exchange kernel (II), $N N$ refers to pole determined from elastic channel and $\Delta \Delta$ to poles from $\pi \Delta \rightarrow \pi \Delta$. The PDG values are taken from [11].

\begin{tabular}{lcccc}
\hline \hline$R(\mathrm{fm})$ & $\begin{array}{c}\operatorname{Re} W_{p} \\
(\mathrm{MeV})\end{array}$ & $\begin{array}{c}-2 \operatorname{Im} W_{p} \\
(\mathrm{MeV})\end{array}$ & $|r|(\mathrm{MeV})$ & $\vartheta$ \\
\hline PDG & 1210 & 100 & 46 & $-46^{\circ}$ \\
$0.123(\mathrm{I})$ & 1205 & 94 & 44 & $-56^{\circ}$ \\
$0.200(\mathrm{II})$ & 1203 & 98 & 47 & $-55^{\circ}$ \\
PDG & 1510 & 270 & 25 & $-180^{\circ}$ \\
Ref. [18] & 1469 & 314 & 38 & $173^{\circ}$ \\
$0.200(\Delta \Delta)$ & 1376 & 255 & 10.6 & $-153^{\circ}$ \\
$0.800(\Delta \Delta)$ & 1379 & 219 & 15.4 & $-162^{\circ}$ \\
$0.800(N N)$ & 1380 & 163 & 8.0 & $-107^{\circ}$ \\
\hline \hline
\end{tabular}

The singular value decomposition mentioned in the previous section allows us to extract the probabilities for the $\pi N$ and $\pi \Delta$ intermediate states in the scattering amplitudes as a function of $W$ (normalized to unity). The probability for the $\pi \Delta$ component in elastic scattering is displayed in Fig. 4 for four different $R$. Similar behavior is observed also upon inclusion of the resonant state discussed in the next subsection. Just about the $\pi N$ threshold the $\pi N$ component is dominant, while in the region around and above $1400 \mathrm{MeV}$ the $\pi \Delta$ component starts to dominate. This explains the constancy of width of the second resonance, which primarily depends on the width of the outgoing $\Delta$ (which is assumed to be constant in the present calculation).

\section{B. Including the resonant state at $1232 \mathrm{MeV}$}

We now turn to a more realistic model introducing a genuine three-quark resonant state. In order to be able to identify the most relevant degrees of freedom in the physically interesting region below $1700 \mathrm{MeV}$, we work with only two channels, the $\pi N$ and the $\pi \Delta$. We adjust the bare mass by fixing the Breit-Wigner resonance mass (i.e., the zero of $\operatorname{Re} T$ )

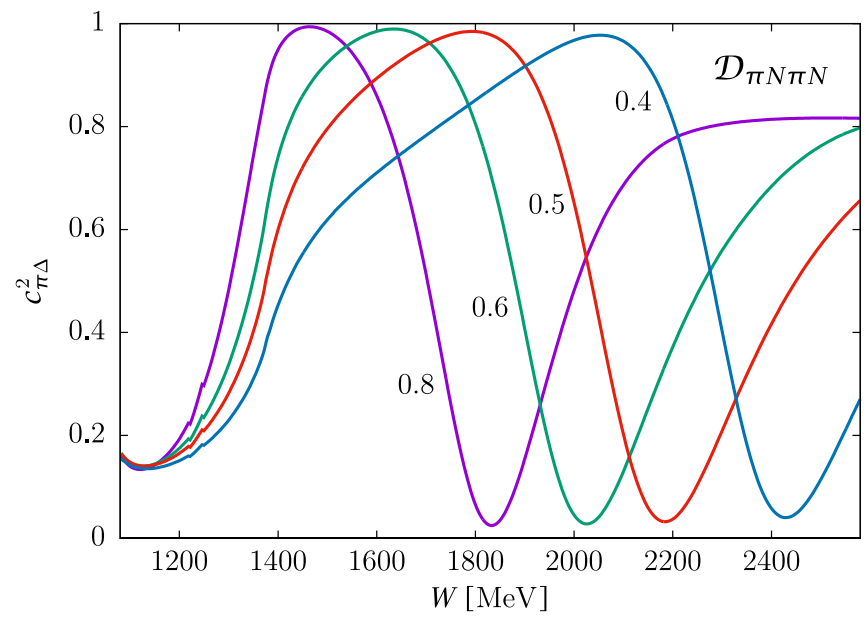

FIG. 4. The probability for the $\pi \Delta$ component in the $\mathcal{D}_{\pi N \pi N}$ amplitude as a function of $W$ for four different $R$ (in $\mathrm{fm}$ ).

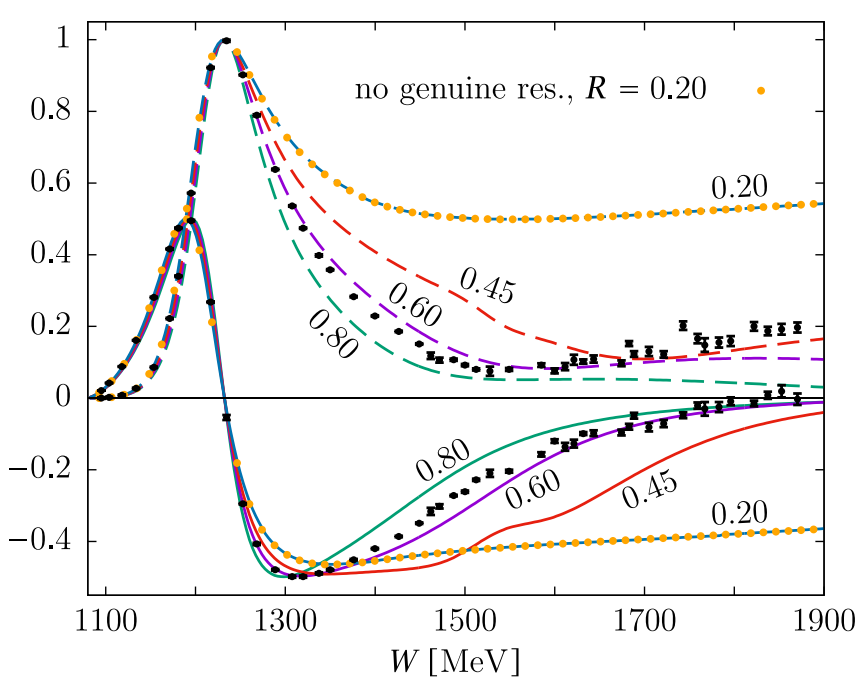

FIG. 5. $T$-matrix amplitudes with and without included genuine resonance at $1232 \mathrm{MeV}$ for $R=0.8,0.60,0.45 \mathrm{fm}$ and $0.200 \mathrm{fm}$. Experimental data are from $[28,29]$.

to $1232 \mathrm{MeV}$ and further fix the bare $\pi N \Delta$ coupling constant to $110 \%$ of the quark-model value and the bare $\pi \Delta \Delta$ coupling constant to $55 \%$ in order to (partially) compensate for the channels not taken into account.

In addition to Eq. (9) we now solve Eq. (8); both equations involve the same kernel, which is identical to the one of the purely dynamical model, and hence the behavior of $w_{\min }(W)$ coincides with that shown in Fig. 1 . The scattering amplitudes are displayed in Fig. 5 for four different $R$. The experimental amplitudes are best reproduced for $R$ between $R=0.8 \mathrm{fm}$ and $R=0.6 \mathrm{fm}$, while at $R=0.45 \mathrm{fm}$ a structure at around $W=1500$ starts to become visible. For $R=0.20$ fm the amplitudes coincide with those of the purely dynamical model. Analyzing the emergence and evolution of poles in the complex plane in Fig. 6, we notice that the position of the lowest pole corresponding to $\Delta(1232)$ stays close

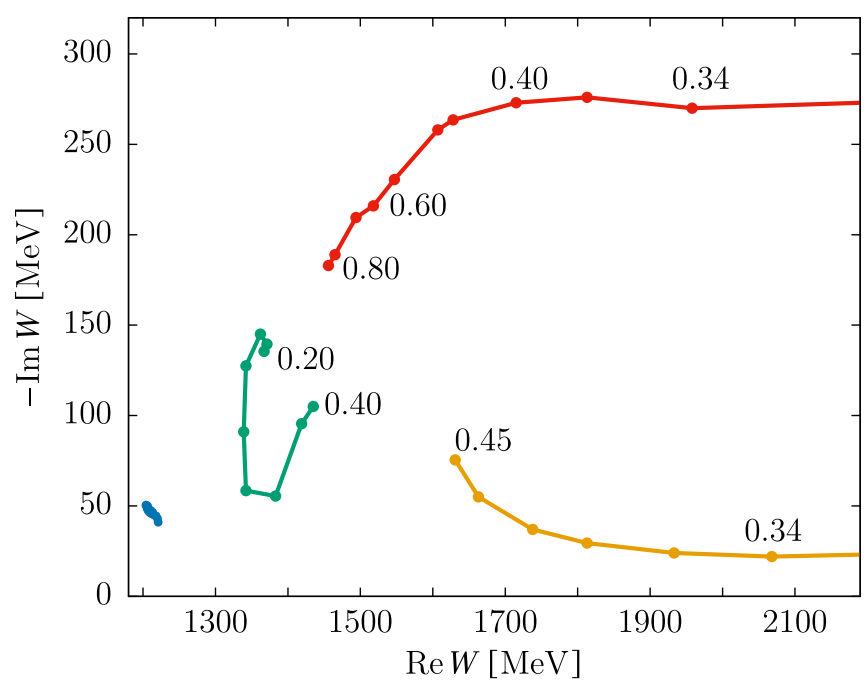

FIG. 6. Evolution of poles in the complex plane as a function of $R$ (in $\mathrm{fm}$ ) with included genuine resonance at $1232 \mathrm{MeV}$. 
TABLE II. $S$-matrix pole position, modulus, and phase with one resonant state for $R=0.8,0.6,0.2 \mathrm{fm}$, and with an additional resonant state with a bare mass 2.2 and $2.0 \mathrm{GeV}$. The PDG values are taken from [11].

\begin{tabular}{lcccr}
\hline \hline$R(\mathrm{fm})$ & $\begin{array}{c}\operatorname{Re} W_{p} \\
(\mathrm{MeV})\end{array}$ & $\begin{array}{c}-2 \operatorname{Im} W_{p} \\
(\mathrm{MeV})\end{array}$ & $|r|(\mathrm{MeV})$ & $\vartheta$ \\
\hline PDG & 1510 & 270 & 25 & $-180^{\circ}$ \\
Ref. [18] & 1469 & 314 & 38 & $173^{\circ}$ \\
0.800 & 1449 & 350 & 26.3 & $174^{\circ}$ \\
0.600 & 1508 & 427 & 50.0 & $-165^{\circ}$ \\
0.200 & 1367 & 271 & 12.1 & $-160^{\circ}$ \\
$m_{\Delta^{*}}=2.2 \mathrm{GeV}$ & & & & \\
0.800 & 1453 & 360 & 44 & $-174^{\circ}$ \\
0.600 & 1570 & 397 & 66 & $-166^{\circ}$ \\
$m_{\Delta^{*}}=2.0 \mathrm{GeV}$ & & & & \\
0.800 & 1452 & 347 & 46 & $-179^{\circ}$ \\
0.600 & 1631 & 340 & 68 & $-140^{\circ}$ \\
\hline \hline
\end{tabular}

to the standard PDG value and only moves toward slightly larger widths and smaller masses for very small $R$. There is a second branch starting with masses around $1450 \mathrm{MeV}$ and widths of $350 \mathrm{MeV}$ evolving towards higher values for both quantities. At smaller values, below $R$ at which $w_{\min }$ crosses zero, a third branch emerges starting with a small residue and evolving toward the second pole of the purely dynamical model. There is a fourth branch essentially identical to the third branch discussed in the previous section.

We conclude this section by noting that the main effect of including a bare resonant $\Delta$ state is the mixing of the bare state with the dynamically generated states, which results in pushing the dynamically generated resonance obtained in the previous section towards higher energies; for larger $R$ its properties come closer to the values in the PDG table (see Table II) and it can be identified as the $\Delta(1600)$ resonance.

\section{Including the second resonant state}

We finally consider the inclusion of the second genuine three-quark resonant state in which one quark is promoted to the $2 s$ state. In our previous work on the $N(1440)$ we found a strong mixing of such a configuration with the dynamically generated state; it is therefore important to check whether such a mixing plays a sizable role also in the P33 partial wave.

As discussed in the Introduction, we expect that a bare state with such a configuration does not exist below $2000 \mathrm{MeV}$. We therefore consider two possible bare masses of 2200 and $2000 \mathrm{MeV}$. We used the pion coupling constants obtained in the cloudy bag model without any further adjustment: for $\pi N R$ and $\pi \Delta R$ the constants are $46 \%$ of the corresponding quark-model values for the $(1 s)^{3}$ configuration. We assume the same bag radius for both states.

The calculation proceeds by introducing an additional term $c_{\gamma R^{*}} \mathcal{V}_{\alpha R^{*}}(k)$ in (4) with $\mathcal{V}_{\alpha R^{*}}(k)$ satisfying an analogous equation to Eq. (8). The equations for $c_{\gamma R}$ and $c_{\gamma R^{*}}$, however,

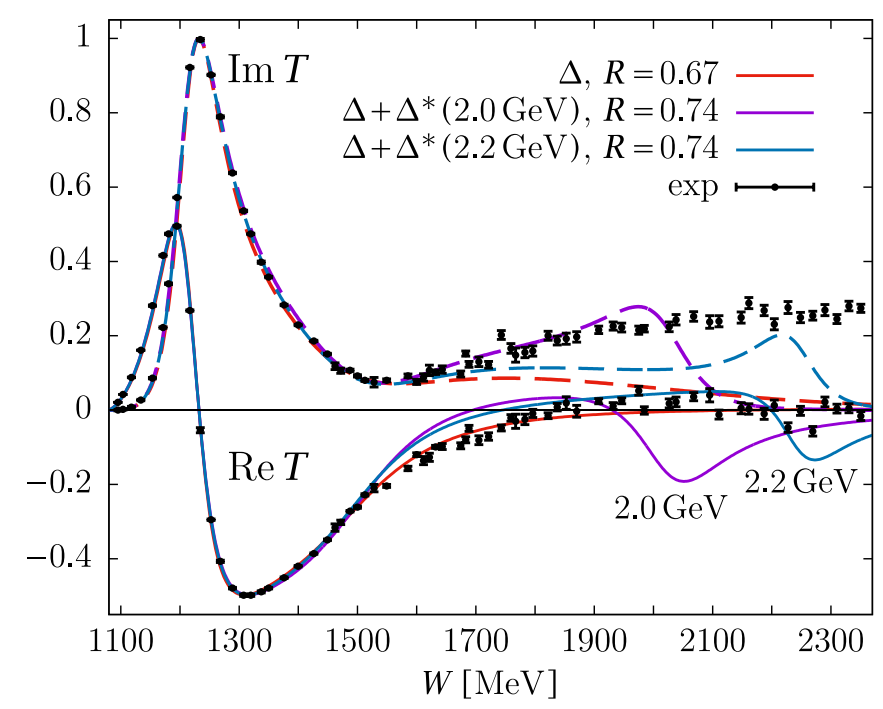

FIG. 7. $T$-matrix amplitude with the included resonant $\Delta$ state for $R=0.67 \mathrm{fm}$, and the amplitude with the included second state at bare masses 2000 and $2200 \mathrm{MeV}$ for $R=0.74 \mathrm{fm}$. Experimental data are from [28,29].

become more complicated due to mixing of the bare resonances through pion loops, as described in [8]. We fix again the Breit-Wigner mass of the lower resonance to $1232 \mathrm{MeV}$ by adjusting the bare $\Delta$ mass; this procedure works, provided that the bare $\Delta$ mass does not come too close to the bare mass of the upper states, which occurs around $R=0.45 \mathrm{fm}$. The resulting Breit-Wigner mass of the upper resonance remains close to its bare value even for smaller $R$ where the two bare state strongly mix.

Figure 7 displays the $T$-matrix amplitude with and without including the second resonant state for the bag radii for which the amplitude agrees best with experiment. While the imaginary part of the amplitude is improved in the intermediate regime by introducing the second state, a typical resonant behavior emerging around $2000 \mathrm{MeV}$ for the bare mass of $2000 \mathrm{MeV}$ is not supported by experiment. It seems therefore that such a low mass is ruled out. Adding further channels may enhance the amplitude and improve the agreement and may eventually support a bare mass as low as $2200 \mathrm{MeV}$. We shall nonetheless include both bare states in our further analysis.

The evolution of the poles pertinent to the second and the third resonances is compared to the evolution of the second resonance discussed in the previous subsection in Fig. 8. We notice that the presence of the new resonant state affects little the properties for larger $R$ but already for $R=0.60 \mathrm{fm}$ the mass of the resonance is further increased with respect to the purely dynamical resonance as well as the resonance with a single resonant state (see also Table II). The third resonance pole, starting on the real axis at the bare mass of the resonant state, evolves towards large $-\operatorname{Im} W$ almost in a straight line and does not bend toward lower masses as in the case of the $N(1440)$ resonance. This indicates that the excited quark configuration plays a rather insignificant role in the formation of the $\Delta(1600)$ resonance (in the physically sensible range of radii). 


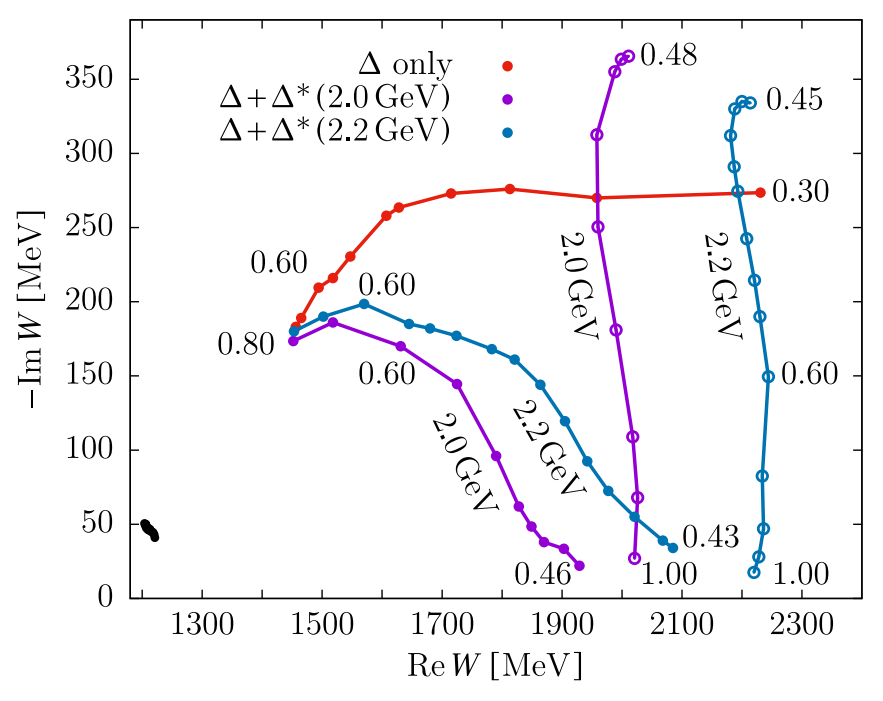

FIG. 8. Evolution of the poles pertinent to the second resonance (full circles) and the third resonance (open circles) in the complex plane, as a function of $R$ (in $\mathrm{fm}$ ).

\section{ELECTROPRODUCTION AMPLITUDES}

Electroproduction is an important tool to study the resonance structure. In particular, the extracted $Q^{2}$ dependence of helicity amplitudes may reveal the spatial distribution of quark and meson degrees of freedom. As an example, let us mention our early calculation of the helicity amplitudes in the case of the $\Delta(1232)$ resonance [30], in which we have been able to disentangle the quark and the pion degrees of freedom and have shown that the latter strongly dominate the $E_{1+}$ production amplitude; similarly, studying the structure of the $N(1440)$ resonance [23], we have been able to explain the zero crossing of the $A_{1 / 2}$ amplitude by its transition from the pion-dominated region at low $Q^{2}$ to the region dominated by quarks at larger $Q^{2}$. This type of calculation is based on the assumption that it is possible to extract the resonant state from (1) and calculate the electromagnetic (EM) part of the electroproduction amplitude by evaluating the corresponding EM multipole between the ground state and the resonant state. Such an approach is justified if the resonance is sufficiently narrow and can be separated from the background and possible neighboring resonances. This is certainly not fulfilled in the case of $\Delta(1600)$. It seems that the only physically sensible way to obtain the helicity amplitudes is to extract them from the electroproduction amplitudes at the $S$-matrix pole for different values of $Q^{2}$.

The calculation is organized as follows: the $K$ matrix acquires a new channel, $\gamma N$, and the corresponding matrix elements are calculated from (2) with the EM interaction,

$$
V_{\mu}^{\gamma}\left(\boldsymbol{k}_{\gamma}\right)=\frac{e_{0}}{\sqrt{2 \omega_{\gamma}}} \int d \boldsymbol{r} \boldsymbol{\varepsilon}_{\mu} \cdot \boldsymbol{j}(\boldsymbol{r}) e^{i \boldsymbol{k}_{\gamma} \cdot \boldsymbol{r}},
$$

replacing the strong one. Here $\boldsymbol{k}_{\gamma}$ and $\mu$ are the momentum and the polarization of the incident photon, and the current involves the quark and the pion part:

$$
\boldsymbol{j}(\boldsymbol{r})=\bar{\psi} \gamma\left(\frac{1}{6}+\frac{1}{2} \tau_{0}\right) \psi+i \sum_{t} t \pi_{t}(\boldsymbol{r}) \nabla \pi_{-t}(\boldsymbol{r}) .
$$

TABLE III. Photoproduction residues at the pole of $\Delta(1600)$. The values of the modulus should be divided by 1000 .

\begin{tabular}{lcccc}
\hline \hline & $\left|\operatorname{Res} E_{1+}\right|$ & $\theta_{E}$ & $\left|\operatorname{Res} M_{1+}\right|$ & $\theta_{M}$ \\
\hline$\gamma N \rightarrow \pi N$ & & & & \\
Ref. [20] & 0.44 & $127^{\circ}$ & 2.53 & $-149^{\circ}$ \\
$R=0.8 \mathrm{fm}$ & 0.30 & $143^{\circ}$ & 2.02 & $173^{\circ}$ \\
$R=0.6 \mathrm{fm}$ & 0.49 & $115^{\circ}$ & 4.46 & $122^{\circ}$ \\
$\gamma N \rightarrow \pi \Delta$ & & & & \\
Ref. [32] & $2 \pm 1$ & $30^{\circ} \pm 30^{\circ}$ & $12 \pm 3$ & $65^{\circ} \pm 25^{\circ}$ \\
$R=0.8 \mathrm{fm}$ & 0.41 & $158^{\circ}$ & 6.51 & $119^{\circ}$ \\
$R=0.6 \mathrm{fm}$ & 0.67 & $158^{\circ}$ & 13.3 & $127^{\circ}$ \\
\hline \hline
\end{tabular}

Only $M 1$ and $E 2$ multipoles contribute in the $P 33$ partial wave. The $M_{1+}$ and $E_{1+}$ amplitudes (see Fig. 9 for $E_{1+}$ ) are calculated and the respective residues are determined by using the $\mathrm{L}+\mathrm{P}$ expansion. From these residues the helicity amplitudes (photodecay amplitudes) $A_{h}^{\text {pole }}$ are calculated as [31]

$$
A_{h}^{\text {pole }}=\sqrt{\frac{16 \pi k_{\pi} M_{p}}{3 k_{\gamma} m_{N} \operatorname{Res}_{\pi N}}} \operatorname{Res} \mathcal{A}_{1+}^{h},
$$

valid for the $P 33$ partial wave. Here $k_{\pi}$ and $k_{\gamma}$ are the pion and photon momenta evaluated at the pole, $M_{p}$ is the mass of the resonance, and $\operatorname{Res}_{\pi N}$ the elastic $\pi N$ residue; $\mathcal{A}_{1+}^{1 / 2}=$ $-\frac{1}{2}\left(M_{1+}+3 E_{1+}\right)$ and $\mathcal{A}_{1+}^{3 / 2}=-\frac{\sqrt{3}}{2}\left(M_{1+}-E_{1+}\right)$.

In the first step we calculate the photoproduction amplitudes and compare them to the residues obtained by Švarc et al. [20] for $\gamma N \rightarrow \pi N$ and of Sokhoyan et al. [32] for $\gamma N \rightarrow \pi \Delta$; see Table III.

In the next step we calculate the $Q^{2}$ dependence of the helicity amplitudes $A_{1 / 2}$ and $A_{3 / 2}$ at the pole by first calculating the electroproduction amplitudes as a function of $W$ at finite $Q^{2}$ and then determine the residue of the pole for each

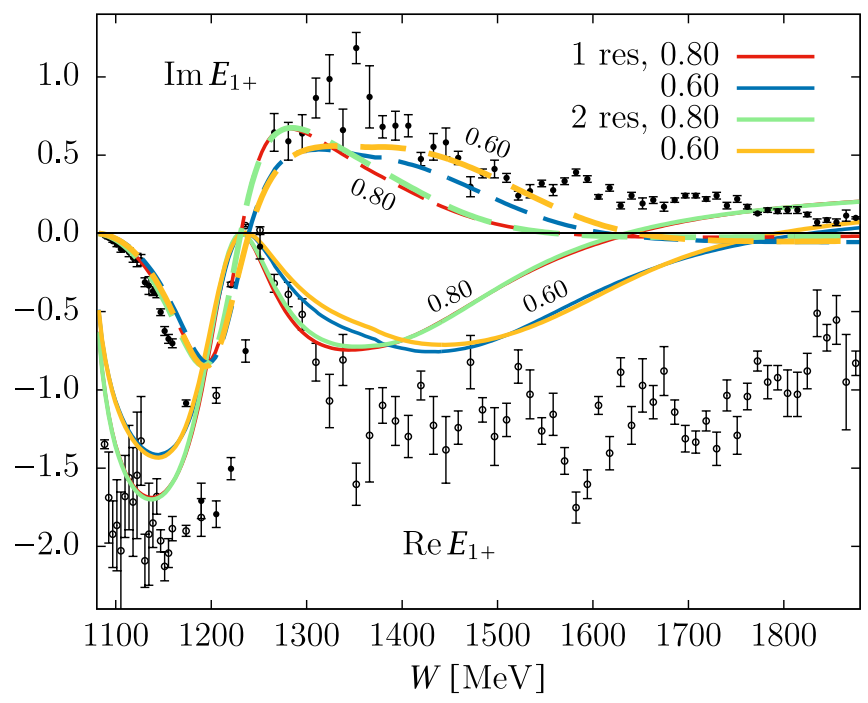

FIG. 9. Photoproduction $E_{1+}$ amplitude (in $\mathrm{mfm}$ ) for $R=0.6$ and $0.8 \mathrm{fm}$ evaluated in the model with one or two resonant threequark states, the second one at $m_{\Delta *}=2.2 \mathrm{GeV}$. Experimental data are from $[28,29]$. 
TABLE IV. Helicity amplitudes at the photon point in units of $10^{-3} \mathrm{GeV}^{-1 / 2}$.

\begin{tabular}{lcccc}
\hline \hline & $\left|A_{1 / 2}^{\text {pole }}\right|$ & $\theta_{1 / 2}$ & $\left|A_{3 / 2}^{\text {pole }}\right|$ & $\theta_{3 / 2}$ \\
\hline Ref. [32] & $53 \pm 10$ & $(130 \pm 15)^{\circ}$ & $55 \pm 10$ & $(152 \pm 15)^{\circ}$ \\
Ref. [12] & 193 & $15^{\circ}$ & 254 & $175^{\circ}$ \\
Ref. [34] & $53 \pm 10$ & $(130 \pm 25)^{\circ}$ & $41 \pm 11$ & $(165 \pm 17)^{\circ}$ \\
Ref. [35] & 72 & $-109^{\circ}$ & 136 & $-98^{\circ}$ \\
$R=0.8 \mathrm{fm}$ & 44 & $-104^{\circ}$ & 47 & $-89^{\circ}$ \\
$R=0.6 \mathrm{fm}$ & 68 & $22^{\circ}$ & 79 & $25^{\circ}$ \\
\hline \hline
\end{tabular}

$Q^{2}$ separately by using the $\mathrm{L}+\mathrm{P}$ expansion. The resulting amplitudes are displayed in Fig. 10 and compared to the phenomenological amplitudes of the MAID2007 analysis [33] evaluated at $W=1232 \mathrm{MeV}$ and at $1470 \mathrm{MeV}$. One should keep in mind that the latter amplitudes have been evaluated by assuming a Breit-Wigner behavior for the resonance, while ours are extracted from the pole residue. It is known that the quark contribution to the magnetic amplitudes is considerably underestimated in our model, particularly at smaller $Q^{2}$, due to the fact that the spinors are limited to the interior of the bag. We expect that this effect is present also in the amplitudes pertinent to the $\Delta(1600)$ resonance. The helicity amplitudes roughly follow the same trend for both resonances, similar to the MAID amplitudes. At low $Q^{2}$ there is, however, a substantial difference due to the $E 2$ multipole, which is considerably larger than in the phenomenological parametrization; as a result, the $A_{1 / 2}$ amplitude at the photon point becomes almost equal to the $A_{3 / 2}$. Taking into account the simplicity of the quark model embedded into our coupled-channels framework as well as large uncertainty of the experimental data regarding this resonance, our values compare favorably to the values from Refs. [32,34] (see Table IV). Since the E2 multipole contribution originates entirely from the pion current, this effect is a strong signal of the important role of the pion cloud in the $\Delta(1600)$ and supports our picture of the resonance.

Let us stress that our model reproduces reasonably well the $E_{1+}$ photoproduction amplitude (Fig. 9) in the physically relevant range of $W$, which speaks in favor of our model. The figure also shows that the presence of the second baryon state at $2.2 \mathrm{GeV}$ has little effect, particularly for larger $R$.

\section{CONCLUSION}

We have studied the mechanism of resonance formation in the $P 33$ partial wave in a model including only the $\pi N$ and the $\pi \Delta$ channels. The $\mathrm{L}+\mathrm{P}$ expansion has been used to extract the $S$-matrix resonance-pole parameters. We have shown that assuming only the $u$-channel exchange diagrams, the system supports two resonances of dynamical origin, the lowest one with a pole mass around $1200 \mathrm{MeV}$, dominated by the $\pi N$ loops, and the second one, dominated by the $\pi \Delta$ loops, with a mass slightly below $1400 \mathrm{MeV}$. For a sufficiently large cutoff parameter, the position and the residue almost perfectly agree with the PDG values, yet the corresponding size of the quark core appears to be much too small to make such a model realistic.

Including a genuine three quark resonant state in the $s$ channel, it becomes possible to reproduce sufficiently well the scattering data in the intermediate energy region by using physically sensible values for the cutoff. The properties of the $\Delta(1232)$ are well reproduced and, furthermore, the second dynamically generated resonance is pushed toward a somewhat higher pole mass of around $1500 \mathrm{MeV}$, acquiring a width which agrees with still rather uncertain PDG values for the $\Delta(1600)$ resonance.

We have checked whether the inclusion of the $s$-wave $\sigma$ meson as well as the quark configuration with one quark excited to the $2 s$ state-which has turned out to play a dominant role in the case of the $N(1440)$ resonance-may change the above picture. The $\sigma \Delta$ channel, as the counterpart of the $\sigma N$ channel in the $P 11$ partial wave, starts to influence the results only above $1700 \mathrm{MeV}$, while the evolution of the pole stemming from the leading $(1 s)^{2} 2 s$ configuration is well separated from the pole evolution pertinent to the second dynamically generated resonance. The excited quark core configuration could eventually be the dominant ingredient in one of the higher $P 33$ resonances.

Electroproduction in the energy region below $\approx 1700 \mathrm{MeV}$, particularly the extraction of helicity amplitudes at finite $Q^{2}$, seems to be the most decisive test to confirm the validity of our picture of the $\Delta(1600)$ resonance. Our model predicts a relatively strong contribution from the $E 2$ multipole originating solely from the photon interaction with the pion cloud and dominating at small $Q^{2}$ due to the large extent of the pion field. As a result, $A_{1 / 2}$ is enhanced, while $A_{3 / 2}$ is diminished with
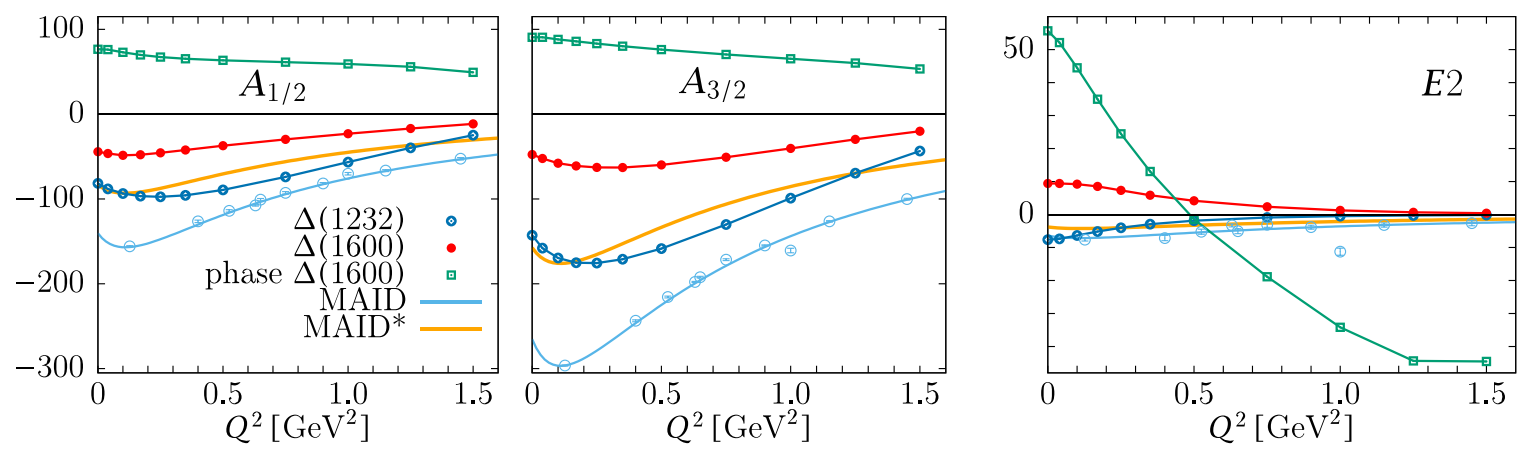

FIG. 10. Helicity amplitudes (units of $10^{-3} \mathrm{GeV}^{-1 / 2}$, phase in degrees) at the $S$-matrix pole of the $\Delta(1232)$ and $\Delta(1600)$ resonances for $R=0.8 \mathrm{fm}$, and the corresponding multipole amplitude $E 2$, compared to the phenomenological parametrization [33]. 
respect to their ratio of $\sqrt{3}$, assuming $M 1$ dominance, and the two amplitudes in fact become comparable in size. Furthermore, we predict that they drop smoothly to zero at large $Q^{2}$ and do not exhibit a zero crossing, as is the case with the $A_{1 / 2}$ amplitude in the Roper resonance, where the quark $(1 s)^{2} 2 s$ configuration is strong and produces a contribution with the opposite sign with respect to the pion cloud contribution.

We conclude that the $\Delta(1600)$ is perhaps the most clean example of a dynamically generated nonstrange resonance in the second and third resonance regions.
[1] H. L. Anderson, E. Fermi, E. A. Long, and D. E. Nagle, Phys. Rev. 85, 934 (1952).

[2] H. L. Anderson, E. Fermi, E. A. Long, and D. E. Nagle, Phys. Rev. 85, 936 (1952).

[3] G. F. Chew and F. E. Low, Phys. Rev. 101, 1570 (1956).

[4] C. B. Lang, L. Leskovec, M. Padmanath, and S. Prelovsek, Phys. Rev. D 95, 014510 (2017).

[5] A. L. Kiratidis, W. Kamleh, D. B. Leinweber, Z. W. Liu, F. M. Stokes, and A. W. Thomas, Phys. Rev. D 95, 074507 (2017).

[6] O. Krehl, C. Hanhart, C. Krewald, and J. Speth, Phys. Rev. C 62, 025207 (2000).

[7] D. Rönchen et al., Eur. Phys. J. A 49, 44 (2013).

[8] B. Golli, H. Osmanović, S. Širca, and A. Švarc, Phys. Rev. C 97, 035204 (2018).

[9] N. Suzuki, B. Juliá-Díaz, H. Kamano, T.-S. H. Lee, A. Matsuyama, and T. Sato, Phys. Rev. Lett. 104, 042302 (2010).

[10] H. Kamano, S. X. Nakamura, and T.-S. H. Lee, and T. Sato, Phys. Rev. C 81, 065207 (2010).

[11] M. Tanabashi et al. (Particle Data Group), Phys. Rev. D 98, 030001 (2018).

[12] D. Rönchen et al., Eur. Phys. J. A 50, 101 (2014).

[13] S. Capstick and B. D. Keister, Phys. Rev. D 51, 3598 (1995).

[14] G. Ramalho and K. Tsushima, Phys. Rev. D 82, 073007 (2010).

[15] I. G. Aznauryan and V. D. Burkert, Phys. Rev. C 92, 035211 (2015).

[16] Y. Lu et al., Phys. Rev. D 100, 034001 (2019).

[17] A. Švarc, M. Hadžimehmedović, H. Osmanović, J. Stahov, L. Tiator, and R. L. Workman, Phys. Rev. C 88, 035206 (2013).
[18] A. Švarc, M. Hadžimehmedović, R. Omerović, H. Osmanović, and J. Stahov, Phys. Rev. C 89, 045205 (2014).

[19] A. Švarc, M. Hadžimehmedović, H. Osmanović, J. Stahov, and R. L. Workman, Phys. Rev. C 91, 015207 (2015).

[20] A. Švarc, M. Hadžimehmedović, H. Osmanović, J. Stahov, L. Tiator, and R. L. Workman, Phys. Rev. C 89, 065208 (2014).

[21] B. Golli and S. Širca, Eur. Phys. J. A 38, 271 (2008).

[22] A. W. Thomas, Adv. Nucl. Phys. 13, 1 (1984).

[23] B. Golli, S. Širca, and M. Fiolhais, Eur. Phys. J. A 42, 185 (2009)

[24] B. Golli, S. Širca, Eur. Phys. J. A 47, 61 (2011).

[25] B. Golli, S. Širca, Eur. Phys. J. A 49, 111 (2013).

[26] B. Golli, S. Širca, Eur. Phys. J. A 52, 279 (2016).

[27] G. H. Golub and C. F. Van Loan, Matrix Computations, 3rd ed. (Johns Hopkins University Press, Baltimore, 1996).

[28] R. A. Arndt, W. J. Briscoe, I. I. Strakovsky, and R. L. Workman, Phys. Rev. C 74, 045205 (2006).

[29] http://gwdac.phys.gwu.edu/analysis/pr_analysis.html.

[30] M. Fiolhais, S. Sirca, and B. Golli, Phys. Lett. B 373, 229 (1996).

[31] R. L. Workman, L. Tiator, and A. Sarantsev, Phys. Rev. C 87, 068201 (2013).

[32] V. Sokhoyan et al., Eur. Phys. J. A 51, 95 (2015).

[33] D. Drechsel, S. S. Kamalov, and L. Tiator, Eur. Phys. J. A 34, 69 (2007).

[34] A. V. Anisovich et al., Eur. Phys. J. A 48, 15 (2012).

[35] H. Kamano, S. X. Nakamura, T.-S. H. Lee, and T. Sato, Phys. Rev. C 88, 035209 (2013). 